\title{
Management of Dysphagia in Head and Neck Cancer Patient during COVID-19 Pandemic: Practical Strategy
}

\author{
Peter $\mathrm{KM} \mathrm{Ku}, \mathrm{MD}^{1}$ \\ ${ }^{1}$ United Christian Hospital and Tseung Kwan O Hospital
}

April 28, 2020

\begin{abstract}
The global pandemic of 2019 Novel Coronavirus Disease (COVID-19) has tremendously altered routine medical service provision and imposed unprecedented challenges to the healthcare system. This impacts patients with dysphagia complications caused by head and neck cancers. As this pandemic of COVID-19 may last longer than SARS in 2003, a practical workflow for managing dysphagia is crucial to ensure a safe and efficient practice to patients and healthcare personnel. This document provides clinical practice guidelines based on available evidence to date to balance the risks of SARS-CoV-2 exposure with the risks associated with dysphagia. Critical considerations include reserving instrumental assessments for urgent cases only, optimizing the non-instrumental swallowing evaluation, appropriate use of PPE, and use of telehealth when appropriate. Despite significant limitations in clinical service provision during the pandemic of COVID-19, a safe and reasonable dysphagia care pathway can still be implemented with modifications of setup and application of newer technologies.
\end{abstract}

Abstract content goes here

\section{Authors}

${ }^{1,2}$ Peter KM Ku, MD

${ }^{3}$ F. Christopher Holsinger, MD

${ }^{2}$ Jason YK Chan, MD

${ }^{1}$ Zenon CW Yeung, MD

${ }^{4}$ Becky YT Chan, MSc

${ }^{2}$ Michael CF Tong, MD

${ }^{3}$ Heather M Starmer, MA CCC-SLP, BCS-S

\section{Authors' Affiliations}

${ }^{1}$ Department of Otorhinolaryngology - Head and Neck Surgery, United Christian Hospital and Tseung Kwan O Hospital, Hong Kong.

${ }^{2}$ Department of Otorhinolaryngology - Head and Neck Surgery, Prince of Wales Hospital, The Chinese University of Hong Kong, Hong Kong

${ }^{3}$ Division of Head and Neck Surgery, Department of Otolaryngology, Stanford University, Palo Alto, CA, USA

${ }^{4}$ Department of Speech Therapy, Prince of Wales Hospital, Hong Kong 


\title{
Correspondence
}

Peter KM Ku, MD

Department of Otorhinolaryngology - Head and Neck Surgery

United Christian Hospital and Tseung Kwan O Hospital

2 Po Ning Lane, Han Hou, Tseung Kwan O

New Territories, HONG KONG SAR

Email: pkukm@yahoo.com

Phone: 852-91995131

Word Count (Text): 3542

\begin{abstract}
The global pandemic of 2019 Novel Coronavirus Disease (COVID-19) has tremendously altered routine medical service provision and imposed unprecedented challenges to the healthcare system. This impacts patients with dysphagia complications caused by head and neck cancers. As this pandemic of COVID-19 may last longer than SARS in 2003, a practical workflow for managing dysphagia is crucial to ensure a safe and efficient practice to patients and healthcare personnel. This document provides clinical practice guidelines based on available evidence to date to balance the risks of SARS-CoV-2 exposure with the risks associated with dysphagia.

Critical considerations include reserving instrumental assessments for urgent cases only, optimizing the noninstrumental swallowing evaluation, appropriate use of PPE, and use of telehealth when appropriate. Despite significant limitations in clinical service provision during the pandemic of COVID-19, a safe and reasonable dysphagia care pathway can still be implemented with modifications of setup and application of newer technologies.
\end{abstract}

\section{KEYWORDS}

Dysphagia, head and neck, coronavirus, COVID-19, management

\section{Introduction}

The worldwide healthcare system has been inundated by a sudden surge of suspected and confirmed cases of the novel coronavirus disease (COVID-19) since the World Health Organization (WHO) declared the global pandemic of this viral illness on March 11, 2020. In just over a month, 1,914,916 confirmed cases of COVID-19 were reported globally with 123,010 deaths by April 14, 20201. This represents a 10-fold increase in confirmed cases and 15 -fold increase in deaths since the declaration of pandemic. While this global pandemic has flooded and imposed unprecedented challenges to the health care system, the global shortage of personal protective equipment (PPE) has created extra burden to frontline health care personnel.

There are 550,000 new cases of head and neck cancer (HNC) registered worldwide per year. ${ }^{2}$ The incidence of HNC is not anticipated to be affected by pandemic of COVID-19. Dysphagia is one of the most common complaints for HNC patients before, during, and after treatment. These patients often require multidisciplinary care by speech-language pathologists and otolaryngologists in dedicated swallowing disorders clinics. A detailed symptom inventory, oromotor examination, clinical swallowing assessment, objective instrumental swallowing studies, swallowing intervention, and monitoring of progress are still essential for these patients during the COVID-19 pandemic. However, given the significant challenges involving transmission risk and limited PPE, alteration to the typical provision of swallowing services must be considered during this outbreak. As this pandemic of COVID-19 may last longer than SARS in 2003, a practical workflow for managing dysphagia is of utmost importance. In these clinical guidelines, we propose a strategic plan 
to facilitate safe practice in dysphagia management for health care personnel and patients with dysphagia, without jeopardizing the standard of care.

\section{Selection of Swallowing Studies}

Fiberoptic endoscopic evaluation of swallowing (FEES) and videofluoroscopic swallowing studies (VFSS; also sometimes called modified barium swallow) are two common instrumental swallowing studies. During FEES examination, a fiberoptic endoscope is passed by the clinician through the nose of the patient into the pharynx, which allows observation of anatomy of the pharynx/larynx, management of saliva and food/liquid boluses, coordination of the pharynx, tongue and larynx during swallowing, and presence of laryngeal penetration or aspiration into the airway. It is commonly performed by speech-language pathologists and otolaryngologists. VFSS utilizes real-time X-ray to assess the different phases of swallowing and swallowing physiology as the bolus passes through the oral cavity, pharynx and upper cervical esophagus using barium impregnated food materials of different consistencies. It allows for observation of swallowing biomechanics and any consequences of dysfunction including penetration and aspiration of food materials into the airway. VFSS is typically performed by radiologists and speech-language pathologists. While FEES and VFSS have their own merits and limitations, both can be used to assess head and neck cancers patients.

While both VFSS and FEES are appropriate tests to utilize in assessment of swallowing, in the context of the COVID-19 outbreak, clinicians need to consider relative risk with these procedures. The higher risk of aerosolization of matter from the nasal passage and nasopharynx with FEES may suggest that VFSS may be the safer option in the current climate. Additionally, some centers may still practice testing of laryngeal sensation by air-pulse stimulator which fires air-pulses in 50 milliseconds duration with pressure from 2 to $10 \mathrm{mmHg}$ to elicit the reflexive twitching of vocal cords before endoscopic evaluation of swallowing. ${ }^{3-4}$ These air-pulses may either create air current in the pharynx or induce cough if the air pressure is high, which may generate droplets and aerosol. In light of the pandemic of COVID-19, suspension of laryngeal sensory testing and FEES examinations should be seriously considered to minimize aerosol generation.

\section{Selection of Personal Protective Equipment}

Recent studies reported the R0 of SARS-CoV-2 ranged from 5.71-7.23 ${ }^{5}$, which is higher than SARS-CoV (2 to 4 ) in $2003 .{ }^{6}$ Therefore, the virulence of COVID-19 is far higher than SARS and may well explain the seemingly uncontrolled pandemic of COVID-19 in many countries. There has been some confusion throughout the health care community in regard to droplet versus aerosol transmission of COVID-19, and subsequently the different levels of PPE required to minimize transmission risk. Aerosol is formed by droplets of smaller sizes, usually smaller than $5 \mu \mathrm{m}$ in diameter, allowing them to remain suspended in the air, to travel longer distances, and and to penetrate face masks with larger pore sizes. High level PPE such as N95 respirators are mandatory with any aerosol generating procedures (AGP). The recent findings of hyposmia, anosmia and dysgeusia in a sizable proportion of confirmed COVID-19 cases may suggest a higher viral load in the nasal cavity/nasopharynx. ${ }^{7}$ Based on the observation of high viral shredding of coronavirus in the nasal passage/nasopharyn $x^{8}$ and anecdotal evidence of increased risk of transmission in the otolaryngology community, use of positive airway power respirators (PAPR) has been advocated for any nasal procedures which generate aerosol. This recommendation would thus apply to FEES exams.

Evidence shows coughing can generate droplets of size from $0.1 \mu \mathrm{m}$ to $100 \mu \mathrm{m}$ which lie in the range of aerosol generation. ${ }^{9}$ Therefore, we can categorize procedures that may induce coughing, such as FEES and VFSS, as AGP and recommend adoption of the highest level of PPE with face shield or goggles, N95 respirator and isolation gown when undertaking these procedures. Face shield can practically provide more coverage to the eyes and face and prevent contamination of the facial skin which is commonly overlooked by most health care personnel during doffing of PPE leading to later transmission of virus through the nose and eyes through hand spread. Careful donning and doffing of isolation gowns is critical to minimize potential contamination.

\section{Screening and Timing of Examination}

FEES and VFSS can trigger sneezing and/or coughing, leading to aerosolization during the procedure. 
The unpublished data of 982 patients attending the combined dysphagia clinic in Prince of Wales Hospital showed that of those with dysphagia following head and neck cancer treatment, nearly $80 \%$ had impaired laryngeal protective reflex. Thus, the incidence of intense coughing during FEES and VFSS may be low in head and neck cancer patients as their nose and pharynx are less sensitive to instrumentation and penetration/aspiration. However, as evidence shows speaking can generate a sufficient amount of droplets to transmit SARS-CoV-2, ${ }^{10}$ and patients are generally not able to wear a face mask during the swallowing evaluation, we should be more conservative when considering these instrumental swallowing procedures. If the condition is not urgent, we suggest postponing any FEES or VFSS for 14 days, as suggested according to the incubation period of COVID-19 in any high-risk patients based on history ( $\mathbf{T}$ ravel, $\mathbf{O}$ ccupation, $\mathbf{C}$ ontact and C lustering phenomenon as TOCC ) and symptomatology such as fever, cough, shortness of breath, and expectorant. It must also be considered that the patient must self-isolate for that 14-day period in order for the healthcare team to be confident of low risk for completion of the swallowing assessment. Additionally, olfactory disturbance has been noticed to occur in high proportion of confirmed cases of COVID-19 (15 to $60 \%$ ) globally, and can be an early or solitary symptom of infection. ${ }^{11}$ With more supporting evidence in our unpublished data on smell loss (47\%) and taste loss (45\%) in COVID-19 confirmed patients, smell and taste disturbance may serve as markers for potential COVID-19 to enhance surveillance in clinic. For patients who require urgent swallowing evaluation in extenuating circumstances (such as acute status change or newly diagnosed aspiration pneumonia), SARS-CoV-2 testing or full aerosol PPE are recommended to minimize transmission risk.

\section{Setup of Examination Environment for Dysphagia Evaluation}

VFSS is preferable to FEES in the current situation of COVID-19 as it does not involve invasive instrumentation during the procedure and the administering clinician (speech-language pathologists, radiologists or otolaryngologists) can maintain a greater distance from the patient while the examination is undertaken. However, it does require patients to be transferred to radiology department. As most radiology departments do not have negative pressure rooms for containment of any airborne particulates during VFSS, the use of IQAir@ HealthPro@ (Incen AG, Switzerland) air-filter with HEPA class H13 filtration system is recommended. The filter is capable of screening $99.97 \%$ of all particles $>0.3$ microns and would be able to filter any micro-droplets and aerosol generated during VFSS during coughing events. In contrast to VFSS, FEES is more portable and can be moved into negative pressure ventilation rooms. Thus, it may be the preferred option for SARS-CoV-2 positive patients or those under investigation if assessment must be performed in an urgent manner. Preferably, FEES would also be performed in a room with setup of IQAir@ air-filter for all patients to reduce environmental contamination by respiratory droplets during the COVID-19 pandemic (Figure 1). Figure 2 summarizes the workflow for instrumental swallowing evaluation in head and neck cancer patients during the COVID-19 pandemic.

\section{Role of Speech-Language-Pathologists and Dysphagia Clinicians in Head and Neck Patients During COIVID-19 Pandemic}

While instrumental assessment of swallowing is should be limited during the COVID-19 pandemic, speechlanguage pathologists (SLP) and dysphagia clinicians (DC) must still find ways to appropriately evaluate and manage patients with suspected dysphagia. Various tools may be implemented to obtain the most objective, comprehensive evaluation possible. The clinical swallowing assessment should include a thorough case history, evaluation of oral motor and laryngeal function, and oral trials of food and liquid. The Mann Assessment of Swallowing Ability - Cancer (MASA-C) ${ }^{12}$ may be utilized to quantify the degree of swallowing impairment, though multiple items may be difficult to capture if conducted through telehealth. When the clinical evaluation is conducted through telehealth, advanced preparation is necessary to ensure the patient has appropriate food and liquid boluses available. Providing the patient with the International Dysphagia Diet Standardization Initiative (IDDSI ${ }^{13}$ diet level descriptions in advance will allow the clinician to better understand the complexity of the boluses administered. Additional considerations for performance of a clinical evaluation through telehealth is use of clear feeding instruments to allow the clinician to best gauge the size of bolus presented, use of food coloring in boluses to increase visibility, and application of colored tape at 
the level of the thyroid cartilage to aid in visualization of laryngeal elevation during the swallow. ${ }^{14}$

A clinical swallowing evaluation, whether in person or via telehealth should be combined with quantitative swallowing measures to minimize the potential for bias. A number of patient-reported outcome (PRO) measures and clinician-rated scales have been validated for use in the head and neck cancer population. The MD Anderson Dysphagia Inventory is a 20-item PRO that can be used to capture the patient's perception of their swallowing difficulties and has been broadly used in the head and neck cancer population and adapted and validated in many languages. ${ }^{15,16}$ Other swallowing specific PROs include the EAT- $10^{17}$, the Sydney Swallow Questionnaire ${ }^{18,19}$ and the Royal Brisbane Hospital Outcome Measure for Swallowing (RBHOMS ${ }^{20}$. In addition to patient reported outcomes, there are several clinician related tools that can be utilized to quantify dysphagia and its outcomes. The Performance Status Scale Head and Neck (PSSHN) ${ }^{21}$ has two items which are routinely administered to quantify dysphagia impact; the normalcy of diet subscale and the eating in public subscale. Further, the Functional Oral Intake Scale (FOIS) ${ }^{22}$ and Food Intake Level Scale $(\text { FILS })^{23}$ can provide additional information about diet level in regard to tube feeding use. This combination of thorough case history, clinical observation, PROs, and clinician rating scales can provide the clinician with needed information to guide recommendations and treatment planning until instrumental assessment becomes more readily available.

It is important for the SLP and DC to recognize the limitations of non-instrumental methods of swallowing evaluation. While agreement between telehealth and in-person clinical swallowing evaluations is good, there are limitations to clinical evaluations, particularly in patients with more severe dysphagia. ${ }^{24}$ Thus, clinicians may need to adopt a more conservative approach to dysphagia management with close attention to potential markers of complications such as increased cough, fever, and weight loss. In those circumstances, the benefits of completing an instrumental evaluation may outweigh the disadvantages. Given higher risks associated with aerosolization and close proximity during FEES exams, the modified barium swallow would be the preferred tool during the COVID-19 outbreak.

In addition to dysphagia diagnostics, swallowing therapy also may need to be adapted during the pandemic. In general, swallowing therapy should be reserved for telehealth whenever possible to minimize transmission risk. There is a paucity of evidence on the benefits of telehealth in dysphagia therapy, but there is some suggestion of improved treatment adherence in patients receiving telehealth in comparison to patient-lead home treatment. ${ }^{25}$ In addition to telehealth, other technology-driven options such as mobile applications may play a role when available. ${ }^{26,27}$ Swallowing therapists should strive to adapt their virtual visits to provide the highest level of care possible. Post-irradiated patients with history of virally mediated nasopharyngeal and oropharyngeal cancer may find these mobile app and telehealth options very beneficial because they are relatively young, independent, knowledgeable, and receptive to the use of technology. Engaging home caregivers and advanced planning of materials needed may help to facilitate treatment sessions. Table 1 summarize the guidelines for telehealth in swallowing management.

\section{Conclusions}

In the global pandemic of COVID-19 disease when the health care system is under unprecedented pressure, any implementation of medical care should be prioritized according to urgency and safety. Dysphagia can be potentially life threatening if left unattended as it may cause aspiration pneumonia or airway obstruction. While we suggest deferring any non-urgent instrumental swallowing studies, particularly in patients considered at high risk for COVID19 based on TOCC and acute respiratory symptoms, screening and assessment of swallowing function can still be possible through telehealth using various non-instrumental methods. Such assessments can help to mitigate risks associated with dysphagia and to triage those patients most in need of instrumental evaluation. Telehealth can also be used to implement swallowing training, for monitoring, and to review progress as well as to engage home caregivers and plan future services. In cases where instrumental assessment is deemed necessary, we advocate for adoption of conservative, high level PPE use to minimize risk to patients and health care providers.

\section{References}


1. World Health Organization. Coronavirus disease 2019 (COVID): situation report 77. Published April 6, 2020. Accessed April 7, 2020. https://www.who.int/docs/default-source/coronaviruse/situation-reports/20200406sitrep-77-covid-19.pdf?sfvrsn=21d1e632_2

2. Fitzmaurice C, Allen C, Barber RM, et al. Global, regional, and national cancer incidence, mortality, years of life lost, years lived with disability, and disability-adjusted life-years for 32 cancer groups, 1990 to 2015: A systematic analysis for the global burden of disease study. JAMA Oncology 2017;3:524-548

3. Aviv JE. Clinical Assessment of Pharyngolaryngeal Sensitivity.Am J Med . 2000 Mar 6;108 Suppl 4a:68S-72S.

4. Ku PK, Vlantis AC, Leung SF, et al. Laryngopharyngeal Sensory Deficits and Impaired Pharyngeal Motor Function Predict Aspiration in Patients Irradiated for Nasopharyngeal Carcinoma. Laryngoscope. 2010 Feb;120:223-8.

5. Tang B, Wang X, Li Q, et al. Estimation of the Transmission Risk of the 2019-nCoV and Its Implication for Public Health Interventions. J Clin Med. 2020 Feb 7;9:462.

6. Patel ZM, Fernandez-Miranda J, Hwang PH, et al. Precautions for endoscopic transnasal skull base surgery during the COVID-19 pandemic. American Academy of otolaryngology-Head and Neck Surgery, coronavirus disease 2019: resources. Published March 23, 2020, Accessed March 27, 2020 https://www.entnet.org/sites/default/files/uploads/covid-19_endosb_lettertoeditor_neurosurgery_update3.23.20.pdf

7. ENT UK. Loss of sense of smell as marker of COVID-19 infection. Published March 21 2020, Accessed April 6, 2020. https://www.entuk.org/sites/default/files/files/Loss\%20of\%20sense\%20of\%20smell\%20as\%20marker\%20

8. Consensus document on the epidemiology of SARS. World Health Organization Wkly Epidemol Rec. 2003;78:373-5. Accessed April 3, 2020 https://www.who.int/csr/sars/en/WHOconsensus.pdf

9. Lindsley WG, Pearce TA, Hudnall JB, et al. Quantity and size distribution of cough-generated aerosol particles produced by influenza patients during and after illnesses. J Occup Environ Hyg. 2012;9:443449.

10. Yu X, Yang R. COVID-19 Transmission Through Asymptomatic Carriers Is a Challenge to Containment. Influenza Other Respir Viruses. 2020 Apr 4. doi: 10.1111/irv.12743.

11. ENT UK. Anosmia as a potential marker of COVID-19 infection - an update. Published April 6, 2020. Accessed April 8, 2020 https://www.entuk.org/anosmia-potential-marker-covid-19-infection-update

12. Carnaby GD, Crary MA Development and validation of a cancer specific swallowing assessment tool: MASA-C. Supportive Care in Cancer . 2014;22:595-602.

13. Cichero JA, Lam P, Steele CM, et al. Development of international terminology and definitions for texture-modified foods and thickened fluids used in dysphagia management: The IDDSI Framework. Dysphagia. 2017;32:293-314.

14. Sharma S, Ward EC, Burns C, Theodoros D, Russell T. Assessing swallowing disorders online: a pilot telerehabilitation study. Telemedicine e-Health. 2011;17:688-95.

15. Chen AY, Frankowski R, Bishop-Leone J, et al. The development and validation of a dysphagia-specific quality-of-life questionnaire for patients with head and neck cancer: the M.D. Anderson dysphagia inventory. Arch Otolaryngol Head Neck Surg . 2001;127:870-6.

16. Zhang LJ, Jiang N, Li Z, et al. Psychometric properties of the Chinese version of the M.D. Anderson Dysphagia Inventory for head and neck cancer patients. Cancer Nurs. 2017;40:E9-16.

17. Wallace KL Middleton S, \& Cook IJ. Development and validation of a self-report symptom inventory to assess the severity of oro-pharyngeal dysphagia. Gastroenterol . 2000;118:678-87.

18. Belafsky PC, Mouadeb DA, Rees CJ, et al. Validity and reliability of the Eating Assessment Tool (EAT-10). Ann Otol Rhinol Laryngol . 2008;117:919-24.

19. Dwivedi RC, St Rose S, Roe JW, et al. Validation of the Sydney Swallow Questionnaire (SSQ) in a cohort of head and neck cancer patients. Oral Oncol . 2010;46:e10-4.

20. Ward EC, Conroy AL. Validity, reliability and responsivity of the Royal Bisbrane hospital outcome measure for swallowing. Asian Pacific J Speech Lang Hear 1999;4:109-129.

21. List MA, D'Antonio LL, Cella DF, et al. The performance status scale for head and neck cancer patients and the functional assessment of cancer therapy-head and neck scale. Cancer . 1996;77:2294-201. 
22. Crary MA, Mann GD, \& Groher ME. Initial psychometric assessment of a functional oral intake scale for dysphagia in stroke patients. Arch Phys Med Rehabil. 2005;86:1516-20.

23. Kunieda K, Ohno T, Fujishima I, Hojo K, Morita T. Reliability and Validity of a Tool to Measure the Severity of Dysphagia: The Food Intake LEVEL Scale. Pain Symptom Manage . 2013;46:201-6.

24. Ward EC, Burns CL, Theodoros DG, Russell TG. Impact of dysphagia severity on clinical decision making via telerehabilitation. Telemed J E Health. 2014;20:296-303.

25. Wall LR, Ward EC, Cartmill B. Adherence to a prophylactic swallowing therapy program during (chemo) radiotherapy: Impact of service delivery model and patient factors. Dysphagia. 2016;32:27992.

26. Starmer HM, Abrams R, Webster K, et al. Feasibility of a mobile application to enhance swallowing therapy for patients undergoing radiation-based treatment for head and neck cancer. Dysphagia . 2018;33:227-33.

27. Constantinescu G, Kuffel K, King B, Hodgetts W, Rieger J. Usability testing of an mHealth device for swallowing therapy in head and neck cancer survivors. Health Informat $J$. 2018;25:1373-82

\section{Hosted file}

COVID_figure1.docx available at https://authorea.com/users/312328/articles/444303-managementof-dysphagia-in-head-and-neck-cancer-patient-during-covid-19-pandemic-practical-strategy

\section{Hosted file}

covid_figure2.docx available at https://authorea.com/users/312328/articles/444303-managementof-dysphagia-in-head-and-neck-cancer-patient-during-covid-19-pandemic-practical-strategy

\section{Hosted file}

covid_table_1 (1).docx available at https://authorea.com/users/312328/articles/444303-managementof-dysphagia-in-head-and-neck-cancer-patient-during-covid-19-pandemic-practical-strategy 